\title{
Bayesian Learning of Climate Sensitivity I: Synthetic Observations
}

\author{
Michael J. Ring, Michael E. Schlesinger \\ Climate Research Group, Department of Atmospheric Sciences, University of Illinois at Urbana-Champaign, Urbana, USA \\ Email: mjring@atmos.uiuc.edu
}

Received June 27, 2012; revised July 29, 2012; accepted August 11, 2012

\begin{abstract}
The instrumental temperature records are affected by both external climate forcings - in particular, the increase of long-lived greenhouse gas emissions - and natural, internal variability. Estimates of the value of equilibrium climate sensitivity - the change in global-mean equilibrium near-surface temperature due to a doubling of the pre-industrial $\mathrm{CO}_{2}$ concentration - and other climate parameters using these observational records are affected by the presence of the internal variability. A different realization of the natural variability will result in different estimates of the values of these climate parameters. In this study we apply Bayesian estimation to simulated temperature and ocean heat-uptake records generated by our Climate Research Group's Simple Climate Model for known values of equilibrium climate sensitivity, $\Delta \mathrm{T}_{2 \mathrm{x}}$, direct sulfate aerosol forcing in reference year $2000, \mathrm{~F}_{\mathrm{ASA}}$, and oceanic heat diffusivity, $\kappa$. We choose the simulated records for one choice of values of the climate parameters to serve as the synthetic observations. To each of the simulated temperature records we add a number of draws of the quasi-periodic oscillations and stochastic noise, determined from the observed temperature record. For cases considering only values of $\Delta T_{2 x}$ and/or $\kappa$, the Bayesian estimation converges to the value(s) of $\Delta \mathrm{T}_{2 \mathrm{x}}$ and/or $\kappa$ used to generate the synthetic observations. However, for cases studying $\mathrm{F}_{\mathrm{ASA}}$, the Bayesian analysis does not converge to the "true" value used to generate the synthetic observations. We show that this is a problem of low signal-to-noise ratio: by substituting an artificial, continuously increasing sulfate record, we greatly improve the value obtained through Bayesian estimation. Our results indicate Bayesian learning techniques will be useful tools in constraining the values of $\Delta \mathrm{T}_{2 \mathrm{x}}$ and $\kappa$ but not $\mathrm{F}_{\mathrm{ASA}}$. In our Group's future work we will extend the methods used here to the observed, instrumental records of global-mean temperature increase and ocean heat uptake.
\end{abstract}

Keywords: Climate Uncertainty; Bayesian Estimation; Internal Variability

\section{Introduction}

Previous work by our Group [1] has sought to constrain values of the equilibrium climate sensitivity, $\Delta \mathrm{T}_{2 \mathrm{x}}$ - the change in global-mean equilibrium near-surface temperature due to a doubling of the pre-industrial $\mathrm{CO}_{2}$ concentration, direct sulfate aerosol forcing, $\mathrm{F}_{\mathrm{ASA}}$, and oceanic heat diffusivity, $\kappa$, based on a comparison of the root-mean-square error difference between the simulated records produced by our Simple Climate Model [2] and the observed instrumental temperature records [3-7] and ocean heat-uptake [8] record.

These optimizations produce only a single value of $\Delta \mathrm{T}_{2 \mathrm{x}}, \mathrm{F}_{\mathrm{ASA}}$ and $\kappa$ for each combination of temperature and heat-uptake records used. If these records contained only the response to external forcings of the climate system then, assuming we had perfect knowledge of these external forcings, we would be able to constrain the values of the climate parameters exactly. However, the instrumental records contain not only the Earth's response to the external climate forcings, but also variability internal to the climate system. This internal variability includes the "quasi-periodic oscillations" (QPOs) [9]climate oscillations that do not have perfectly regular periods and amplitudes - and a random component that may be considered as stochastic noise.

The observed temperature and ocean heat-uptake records contain but a single realization of the natural variability (NV). However, this NV influences the model's estimation of climate parameters. The values obtained by [1] are optimized for the single realization of NV that has occurred in the observed record. Had the realization of $\mathrm{NV}$ been different, the values for the climate parameters obtained by [1] would have been different as well. Our motivation here is to consider different realizations of $\mathrm{NV}$ and the effects these realizations will have on the estimations of climate sensitivity, aerosol forcing and oceanic diffusivity, with the ultimate goal of applying these techniques to the instrumental records. 
Previously our Group [10] used a bootstrap resampling technique [11] to generate a number of proxies of NV. The bootstrap resampling considers the difference between the observed and model-simulated temperatures or oceanic heat uptake. The original residuals are transformed to uncorrelated data, resampled, and transformed back to a sample from the original data with the same autocorrelation as in the original sample.

Here we incorporate a different technique to investigate the uncertainty in estimations of climate sensitivity, aerosol forcing and oceanic diffusivity: Bayesian analysis. The Bayesian estimation method combines observations with model output and prior information on parameter values. It has become an increasingly popular technique for estimation of parameters in climate research. While our ultimate goal is to apply these techniques to the instrumental data, our focus here is to discuss the development of our methods and their application to synthetic data, whose climate parameters are known.

In Section 2 we discuss Bayes' theorem and our application thereof, our use of our Group's Simple Climate Model (SCM), and our generation of different realizations of NV. In Section 3 we present results examining solely one climate parameter at a time. Trials examining multiple climate parameters simultaneously are shown in Section 4. Finally in Section 5 we discuss our results and intentions for future work.

\section{Methods}

Our primary technique is Bayesian estimation - an analysis of probability where Bayes' theorem is used to update the probability estimate for a hypothesis as new information becomes available. As such, Bayesian learning makes use of both an estimation of the likelihood of a proposition based on currently available data, as well as a prior estimation. We also make use of our Group's SCM and a program to generate different realizations of natural variability consistent with those in the observed temperature record and ocean heat-uptake record $[12,13]$.

Since the introduction of the techniques to the climate sciences [14], Bayesian estimation has become an increasingly popular tool for atmospheric scientists [15]. Bayesian analysis has been used to estimate ranges for global climate parameters including equilibrium climate sensitivity, aerosol radiative forcing and oceanic diffusivity [16-20], detection and attribution of observed climate changes [21-23], and expected future warming under emissions scenarios [24]. Bayesian analysis has also been used to combine multi-model projections of simulated global temperature increases [25-27] and probabilities of particular changes in mean surface temperature and precipitation [28-30]. The techniques have also been applied to regional and local scales such as North Pacific sea-surface temperature projections [31], regional pre- cipitation [32] and temperature $[33,34]$ projections, tropical cyclone frequency and track locations [35-46], Arctic ice occurrence [47], and water management, streamflow and flooding [48-52]. Bayesian estimation has also been used in paleoclimate studies using isotope data [53]. This is not an exhaustive list of the applications of Bayesian techniques to the atmospheric sciences, but rather is intended to indicate the recent frequency of application to problems in weather and climate.

Bayes' theorem relates the posterior probability distribution function (PDF) P for a variable $\theta$, given a set of observations $x$, to a prior probability distribution function and the conditional probability $L$ of observations $x$ given information on $\theta$. This latter term is defined as the likelihood function. Mathematically this is expressed as

$$
P(\theta / x)=L(x / \theta) P(\theta) / P(x)
$$

where

$$
P(x)=\int L(x / \theta) P(\theta) \mathrm{d} \theta
$$

serves to normalize the expression such that

$$
0 \leq P(\theta / x) \leq 1
$$

To explain Bayes' theorem more simply, the left-hand side of the first equation above is the probability of $\theta$ assuming $x$ is true. The factor $L$ on the right-hand side, the likelihood function, is the probability of $x$ assuming $\theta$ is true. The factor $P(\theta)$, the prior, is simply the probability distribution function for $\theta$ that we assumed before learning the new information. Hence the posterior PDF, $P(\theta / \mathrm{x})$, is related to the probability of $x$ assuming a given choice of $\theta$ is true based on the current information, weighted by the probability that $\theta$ is true based on the previous information.

We consider trials with variations in one, two, or all three climate parameters. If we choose $i=1,2, \cdots, i_{\max }$ values of $\Delta \mathrm{T}_{2 \mathrm{x}}, j=1,2, \cdots, j_{\max }$ values of $\mathrm{F}_{\mathrm{ASA}}$ and $k=1$, $2, \cdots, k_{\max }$ values of $\kappa$, then we will have $i_{\max } \times j_{\max } \times k_{\max }$ simulated observational records. However we also consider runs looking at only one or two of the climate parameters. In those cases we will simply choose fixed values for the climate parameter(s) not under study and perform our Bayesian iteration on the other parameter(s). These simulated records are produced by prescribing the values of $\Delta \mathrm{T}_{2 \mathrm{x}}, \mathrm{F}_{\mathrm{ASA}}$ and/or $\kappa$ in our SCM and running the model. For a discussion of the SCM and its forcings, see [1].

To begin the Bayesian analysis, we must state a prior. For the cases shown here, we use a uniform prior. We have also tried Gaussian and Jeffreys priors and obtained similar results.

We now describe the procedure used to obtain our likelihood function $L$. The procedure is illustrated conceptually in Figure 1. We first simulate the temperature 

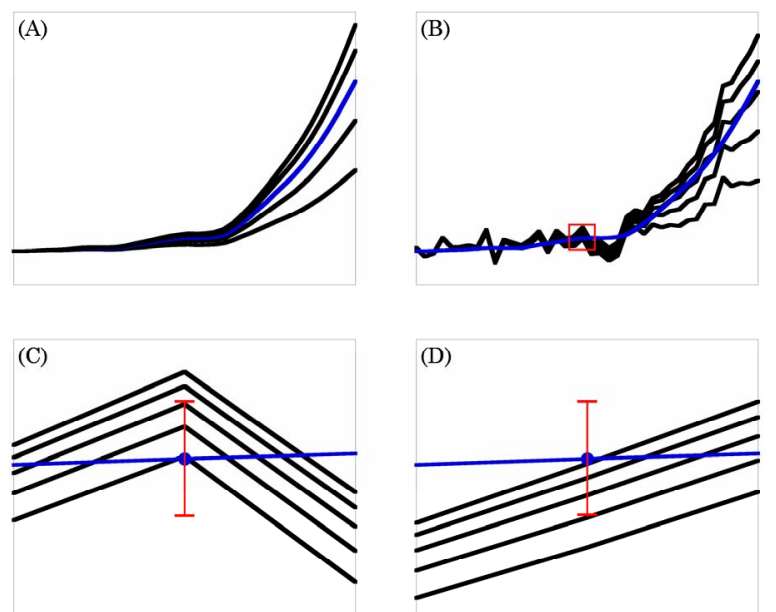

Figure 1. Conceptual illustration of the derivation of the likelihood function. (A) Simulated temperature changes (black) are generated by the SCM for the observed radiative forcing and for multiple prescribed values of climate sensitivity-five here for illustration; (B) One draw of the $\mathrm{NV}$ is taken and added to each simulated temperature change; (C) Close-up of the area enclosed in the red square in (B); (D) The procedure is repeated for another time step and each draw of NV.

change using the SCM for the observed radiative forcing and for multiple prescribed values of the climate parameter(s) to be estimated - climate sensitivity, aerosol radiative forcing in reference year 2000, and ocean diffusivity - and then add a draw of NV (i.e., the QPOs plus stochastic noise) thereto. We do so because the simulated temperature record produced by the SCM contains only the responses to the radiative forcing, but the real temperature records include both the forced response and a realization of NV. In order to extend our model runs past present day, we assume the Representative Concentration Pathways-8.5 (RCP8.5) [54] and its extension as a future emissions scenario. Using this scenario allows us to run the model to 2500 .

These simulated temperature-change records, with NV added, are then compared to the observed temperature changes, either real or synthetic - the latter created by the SCM. At each time step we examine, we ask whether each temperature-change and/or ocean-heat uptake record with $\mathrm{NV}$ added is within a given difference from the observed data-the tolerance. Here the tolerances chosen were $0.01^{\circ} \mathrm{C}$ for temperature and $1 \mathrm{Wm}^{-2}$ for ocean heat uptake, although our results were insensitive to other choices. For cases where we consider two or three of the climate parameters simultaneously, each simulated $+\mathrm{NV}$ record must be within the prescribed tolerance to pass. For example, in a case considering $\Delta \mathrm{T}_{2 \mathrm{x}}$ and $\kappa$, both the simulated + NV global-mean temperature record must be within the tolerance of the synthetic observed globalmean temperature, and the simulated $+\mathrm{NV}$ ocean heat- uptake must be within the tolerance of the synthetic observed ocean heat-uptake, in order to pass.

We generate different expressions of natural variability using Singular Spectrum Analysis [55] and the ARFit package for MATLAB $[12,13]$. We begin by performing SSA on the observed temperature and/or ocean heatuptake record. The leading modes found through SSA combine to form a non-oscillatory trend, and we remove this trend from the record. This leaves the portion of the record attributable to internal variability.

We use the ARFit package to fit the internal variability to an autoregressive model, and then generate $n=1, \cdots$, $n_{\max }$ realizations of $\mathrm{NV}$ with the same autogressive parameters as those found from the SSA analysis. Each of these draws of $\mathrm{NV}$ is added to the simulated records produced by the SCM. Hence, for a three-parameter case where $i_{\max } \times j_{\max } \times k_{\max }$ simulations are made in the SCM, there are now $i_{\max } \times j_{\max } \times k_{\max } \times n_{\max }$ records considered in our Bayesian analysis.

Simulated + NV values within the tolerance-in the case illustrated in Figure 1(C), the first, second and third $\mathrm{NV}$-added temperature changes from the bottom-are considered as having passed at this time step, while the others have not passed. As the likelihood function is normalized over the number of passed draws at each time step, the value of the likelihood function for the case in Figure 1(C) is therefore one-third for simulations + NV 1,2 , and 3 ; and 0 for simulations $+\mathrm{NV} 4$ and 5 . The procedure is repeated for each time step and each draw of NV. For the time step shown in Figure 1(D) the third, fourth, and fifth simulation $+\mathrm{NV}$ from the bottom are passed, so the likelihood function at this time step for this draw of NV is one-third for simulations + NV 3, 4, and 5; and 0 for simulations $+\mathrm{NV} 1$ and 2. Each draw of NV produces a different likelihood function, so we average over many draws of the $\mathrm{NV}$ to obtain our final estimates. In practice, we use 50 values of the climate parameter(s) to simulate the temperature changes and 1000 draws of the NV for each simulated temperature change.

We then go to the first timestep at which we wish to update our Bayesian estimation. For the results shown here, we perform the Bayesian update every fifth year. We then ask, for each value of $\Delta \mathrm{T}_{2 \mathrm{x}}, \mathrm{F}_{\mathrm{ASA}}$, and/or $\kappa$ considered, whether the value of the global temperature change, interhemispheric temperature difference (IHD), or ocean heat uptake, respectively, is within a given tolerance of the value for the case selected as "truth". This analysis gives us the likelihood at each timestep: assuming the value of $\Delta \mathrm{T}_{2 \mathrm{x}}, \mathrm{F}_{\mathrm{ASA}}$, and/or $\kappa$ is true, what is the probability the change in the data is within the prescribed range?

Let us first consider a one-dimensional case, with $i_{\max }$ simulations from the model and $n_{\max }$ draws of noise. For example, for the first simulated NV record $n=1$ and pa- 
rameter $\lambda$, we compute the following, where $\Delta D$ is the change in the data value (for equilibrium climate sensitivity, the change in global-mean temperature) and $\lambda$ is the value of the climate parameter (the equilibrium climate sensitivity):

$$
p_{n=1}(\lambda / \Delta D)=\frac{p_{n=1}(\Delta D / \lambda) p(\lambda)}{p_{n=1}(\Delta D)}
$$

The first term in the numerator on the right-hand side is the likelihood, and the second term in the numerator on the right-hand side is the prior. The term in the denominator serves to normalize the expression.

Since there are $n=1, \cdots, n_{\max }$ realizations of the $\mathrm{NV}$, we generate $\mathrm{n}_{\max }$ realizations of $p_{n}(\lambda / \Delta D)$ at each time step. As each of these $n_{\max }$ realizations of $p_{i}$ is equally likely, we simply average them together to obtain a single posterior at each time step $p_{n}(\lambda / \Delta D)$. That is to say,

$$
p(\lambda / \Delta D)=\frac{\sum_{n=1}^{n_{\max }} p_{n}(\lambda / \Delta D)}{n_{\max }}
$$

This unique final value of the posterior, averaged over all $n_{\max }$ realizations, is then used as the prior for all $n_{\max }$ calculations at the next time step.

For the other climate parameters - direct sulfur forcing, oceanic diffusivity coefficient-we can compute the Bayesian probability similarly, where the data are the IHD or, the oceanic heat uptake, respectively.

For cases examining two or three variables simultaneously, there is an additional step involved. For example, if there are now two parameters under consideration, we must examine two data records, and each data record must be within the tolerance of its "true" simulated record to be counted as a match. This allows us to produce a two-dimensional $L_{n=1}\left(\Delta D_{1}, \Delta D_{2} / \lambda_{1}, \lambda_{2}\right)$ likelihood, the probability of finding both records within the tolerance of the "true" value given that the value of the climate parameters are true.

If we wish to find a univariate likelihood, we must marginalize (integrate) over the variable that we do not wish to consider:

$$
\begin{aligned}
& L_{n=1}\left(\Delta D_{1}, \Delta D_{2} / \lambda_{1}\right)= \\
& \int_{\lambda_{2} \min }^{\lambda_{2} \max } L_{n=1}\left(\Delta D_{1}, \Delta D_{2} / \lambda_{1}, \lambda_{2}\right) p_{n=1}\left(\lambda_{2} / \lambda_{1}\right) \mathrm{d} \lambda_{2} \\
& L_{n=1}\left(\Delta D_{1}, \Delta D_{2} / \lambda_{2}\right)= \\
& \int_{\lambda_{1} \min }^{\lambda_{1} \max } L_{n=1}\left(\Delta D_{1}, \Delta D_{2} / \lambda_{1}, \lambda_{2}\right) p_{n=1}\left(\lambda_{1} / \lambda_{2}\right) \mathrm{d} \lambda_{1}
\end{aligned}
$$

We now have one-dimensional likelihoods for each of our parameters $\lambda_{1}$ and $\lambda_{2}$. We can conclude the Bayesian analysis by combining the likelihood with the prior for each parameter as in Equation (1) above to find the posterior for that particular realization of $\mathrm{NV}$, and average over all the realizations of NV to find the single posterior at each time step as in Equation (2) above, which then becomes the prior in the next time step. A similar procedure is used if all three parameters are considered, but then two marginalizations are required at each time step instead of one.

\section{One-Parameter Results}

In this section we show results using our Bayesian estimation technique considering variations in only one of our three climate parameters $\left(\Delta \mathrm{T}_{2 \mathrm{x}}, \mathrm{F}_{\mathrm{ASA}}, \kappa\right)$. We prescribe a value for the other two parameters in the SCM, and then run the SCM for a number of choices of the parameter under consideration.

In these trials we choose a value of the climate parameter under study and treat the simulation generated therefrom as "truth". That is, we compute PDFs via Bayesian analysis for a parameter whose true value is "known". Since we know the value of the parameter, this allows us to check the correctness of our technique.

We choose $i=1, \cdots, i_{\max }$ values for the climate parameter we wish to study $\left(\Delta \mathrm{T}_{2 \mathrm{x}}, \mathrm{F}_{\mathrm{ASA}}, \kappa\right)$, again choosing single, fixed values for the other two parameters. We run the SCM for each of the $i_{\max }$ values, thereby generating $i_{\max }$ simulated records of global-mean temperature, IHD, or ocean heat uptake. We select one of these trials, and its associated value of the climate parameter, to be the "truth". Since the SCM contains information only of the external forcings, the simulated temperature and ocean heat-uptake records generated will not contain any natural variability, but only the response to the external radiative forcing. In this sense they can be considered "filtered", since they contain no information on either the QPOs or the stochastic noise.

In the figures below we use our calculated PDFs to generate an ensemble of values for each climate parameter at each time step under consideration. We then plot the ensemble of values using box plots. The box spans the 90 percent confidence interval for the parameter under investigation. The dashed lines within the box indicate the 25 th and 75 th percentile for the values, while the solid line within the box is the median. The red horizontal lines indicate the values plus or minus 20 percent of the "true" value for each case.

For one-parameter cases studying $\Delta \mathrm{T}_{2 \mathrm{x}}$ (Figure 2(A)) or $\kappa$ (Figure 2(B)), the Bayesian analysis converges to the "true" value. For each parameter we tried several different choices of "true" value and convergence was obtained similarly in each case. The case in Figure 2(A) begins in 1850 , using a uniform prior for $\Delta \mathrm{T}_{2 \mathrm{x}}$ from $0.5^{\circ} \mathrm{C}$ to $5.5^{\circ} \mathrm{C}$. While little learning occurred in the first 100 years of the simulations, learning occurred thereafter such that, for the trial shown in Figure 2(A), the 90 percent confidence interval converged to the "true" value by 
2125. We also varied the tolerance by which a compareson between the "true" surrogate and the simulated records with natural variability added was counted as a match and found similar results-convergence to the "true" value occurred in each case.

In Figure 2(B) we begin with a uniform prior ranging from $0.30 \mathrm{~cm}^{2} \cdot \mathrm{s}^{-1}$ to $0.79 \mathrm{~cm}^{2} \cdot \mathrm{s}^{-1}$ for the diffusivity. Similar to the results in Figure 2(A), we find learning in the late 20th century and 21 st century such that the 90 percent confidence interval converged to the "true" value by 2150 . As was the case for equilibrium climate sensitivity, the Bayesian estimation for diffusivity converged to the "true" value for different choices of prior, "true" value of the parameter or tolerance interval.

For cases studying the aerosol forcing $\mathrm{F}_{\mathrm{ASA}}$, however, the Bayesian analysis does not converge to the "true" value, even with runs conducted to 2500 . We show one example of this in Figure 3(A). Again, we tested the runs for other values of, and tolerance limits for, $\mathrm{F}_{\mathrm{ASA}}$ and found similar results. The problem is one of a weak signal-to-noise ratio. The RCP/ECP8.5 scenario [54] used here to force the model includes strongly increasing

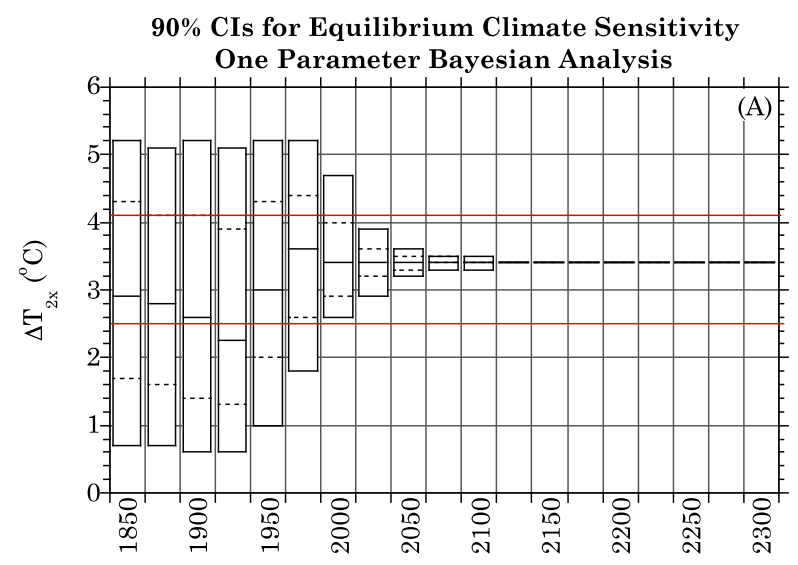

90\% CIs for Diffusivity

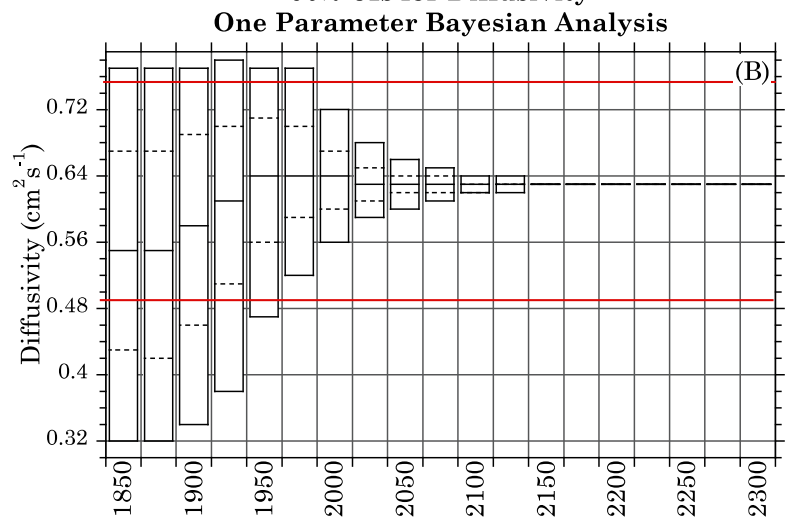

Figure 2. (A) 90 percent confidence intervals for $\Delta T_{2 x}$ using Bayesian estimation technique. "True" value is $3.4^{\circ} \mathrm{C}$. (B) 90 percent confidence intervals for $\kappa$ using Bayesian estimation technique. "True" value is $0.63 \mathrm{~cm}^{2} \cdot \mathrm{s}^{-1}$. long-lived greenhouse-gas forcing, eventually stabilizing at a very high level. Consequently, the global-mean temperature and ocean heat uptake, which the model uses to constrain $\Delta \mathrm{T}_{2 \mathrm{x}}$ and $\kappa$, respectively, continue to increase for several centuries into the future. In contrast, the aerosol forcing contained in the RCP8.5/ECP8.5 scenario does not similarly increase; instead sulfate emissions in the scenario decrease throughout the 21 st century. Therefore the opportunity for learning is reduced, as the future forcing is weak.

To test our hypothesis, we conducted a trial where sulfate emissions were assumed to increase continuously, while the RCP8.5/ECP8.5 forcings were used otherwise. The results for $\mathrm{F}_{\mathrm{ASA}}$ for this trial (Figure 3(B)) show improvement. While the 90 percent CI does not collapse completely to the "true" value as in the cases for $\Delta \mathrm{T}_{2 \mathrm{x}}$ and $\kappa$, it nearly does so and the confidence interval is clearly within 20 percent of the "true" value - a condition that was not satisfied in Figure 3(A). This result was verified for several different choices of "true" value and tolerance limits for comparison between simulated records, and similar behavior was found in all cases.
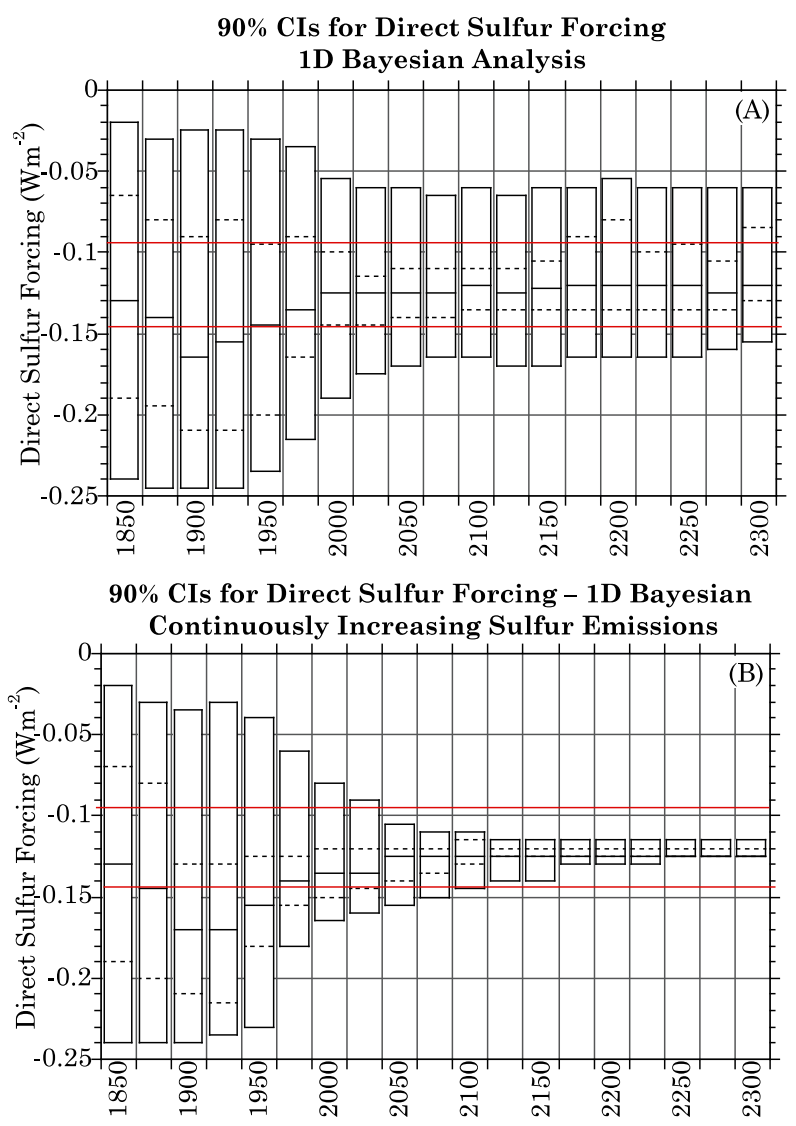

Figure 3. As in Figure 2, but for the direct sulfur aerosol forcing using (A) the RCP8.5 aerosol emissions records or (B) a continuously increasing sulfate emissions record. "True" value is $-0.12 \mathrm{Wm}^{-2}$. 


\section{Multi-Parameter Results}

We have also performed trials using two, or three, of the parameters $\left(\Delta \mathrm{T}_{2 \mathrm{x}}, \mathrm{F}_{\mathrm{ASA}}, \kappa\right)$. Similarly to the one-dimensional results, analyses using only $\Delta \mathrm{T}_{2 \mathrm{x}}$ and $\kappa$ converged to the "true" answer, while those involving $\mathrm{F}_{\mathrm{ASA}}$ in some way did not.

To extend our analyses to multiple dimensions, we must now consider two (or three) of the model outputs. For the two-dimensional case, we select a single value for the climate parameter we are not considering, and hold that value fixed. For the other two parameters, we consider $i=1, \cdots, i_{\max }$ values and $j=1, \cdots, j_{\max }$ values of the second parameter. We then use the SCM to produce these $i_{\max } \times j_{\max }$ simulated observations.

As before we then use SSA analysis to determine the portion of each observed record attributable to internal climate variability, and then use the ARFit package to construct $n=1, \cdots, n_{\max }$ draws of $\mathrm{NV}$ with the same autoregressive properties as those of the observed. We then add each of these NV draws to each of the simulations, so the 2-D Bayesian analysis will consider $i_{\max } \times$ $j_{\max } \times n_{\max }$ records total.

We then turn to our Bayesian estimation. We ask at each timestep we consider whether the value of the simulated record with $\mathrm{NV}$ added is within a tolerance of the value selected as "truth". Since there are now two parameters under consideration, we must examine two data records, and each data record must be within the tolerance of its "true" simulated record to be counted as a match. This allows us to produce a two-dimensional likelihood, $L_{n=1}\left(\Delta D_{1}, \Delta D_{2} / \lambda_{1}, \lambda_{2}\right)$, the probability of finding both records within the tolerance of the "true" value given that the value of the climate parameters are true. We marginalize as described in Section 2 to reduce the two-dimensional likelihood to a single dimension and then continue by using Bayes' theorem.

The behavior in Figures $\mathbf{4}$ and $\mathbf{5}$ is an extension of that seen for trials where only one parameter was considered. In the runs shown in Figure 4, for which a single, fixed value of $F_{A S A}$ was chosen and Bayesian analysis performed on the climate sensitivity and oceanic diffusivity, the uncertainty ranges of both variables found through Bayesian estimation converge to the "true" value. For the case where a single, fixed value of the diffusivity was chosen and the Bayesian analysis performed on climate sensitivity and aerosol forcing (Figure 5), the aerosol forcing estimates fail to converge to the correct value. Other choices of "true" values for the climate parameters produced similar results.

We also performed trials considering all three of the climate parameters $\left(\Delta \mathrm{T}_{2 \mathrm{x}}, \mathrm{F}_{\mathrm{ASA}}, \kappa\right)$ simultaneously. The results of these trials were similar to those of the two-parameter trials: while values of $\Delta \mathrm{T}_{2 \mathrm{x}}$ and $\kappa$ could be properly constrained by the Bayesian analysis, those of
$\mathrm{F}_{\mathrm{ASA}}$ could not.

\section{Conclusions}

From our preliminary results, we have found that our Bayesian estimation procedure works for $\Delta \mathrm{T}_{2 \mathrm{x}}$ and $\kappa$. This has been verified by using synthetic (simulated) observational records, in lieu of the observed data, in the estimation of these quantities. The Bayesian estimation converges to the known values of $\Delta \mathrm{T}_{2 \mathrm{x}}$ and $\kappa$ used to construct the synthetic observations. This finding has been replicated for different choices of $\Delta \mathrm{T}_{2 \mathrm{x}}$ and $\kappa$. In contrast, our Bayesian estimation technique has not been successful in finding the value of $\mathrm{F}_{\mathrm{ASA}}$. We have shown that the problem arises from a low signal-to-noise ratio for the interhemispheric temperature difference used to constrain $\mathrm{F}_{\mathrm{ASA}}$.

For $\Delta \mathrm{T}_{2 \mathrm{x}}$ and $\kappa$ there is a very strong signal, as the RCP8.5/ECP8.5 scenario [54] contains strongly increasing $\mathrm{CO}_{2}$ emissions into the 22nd century. This aggressive emissions scenario results in a concentration of $\mathrm{CO}_{2}$ of over $1900 \mathrm{ppmv}$ in the atmosphere and therefore a strong
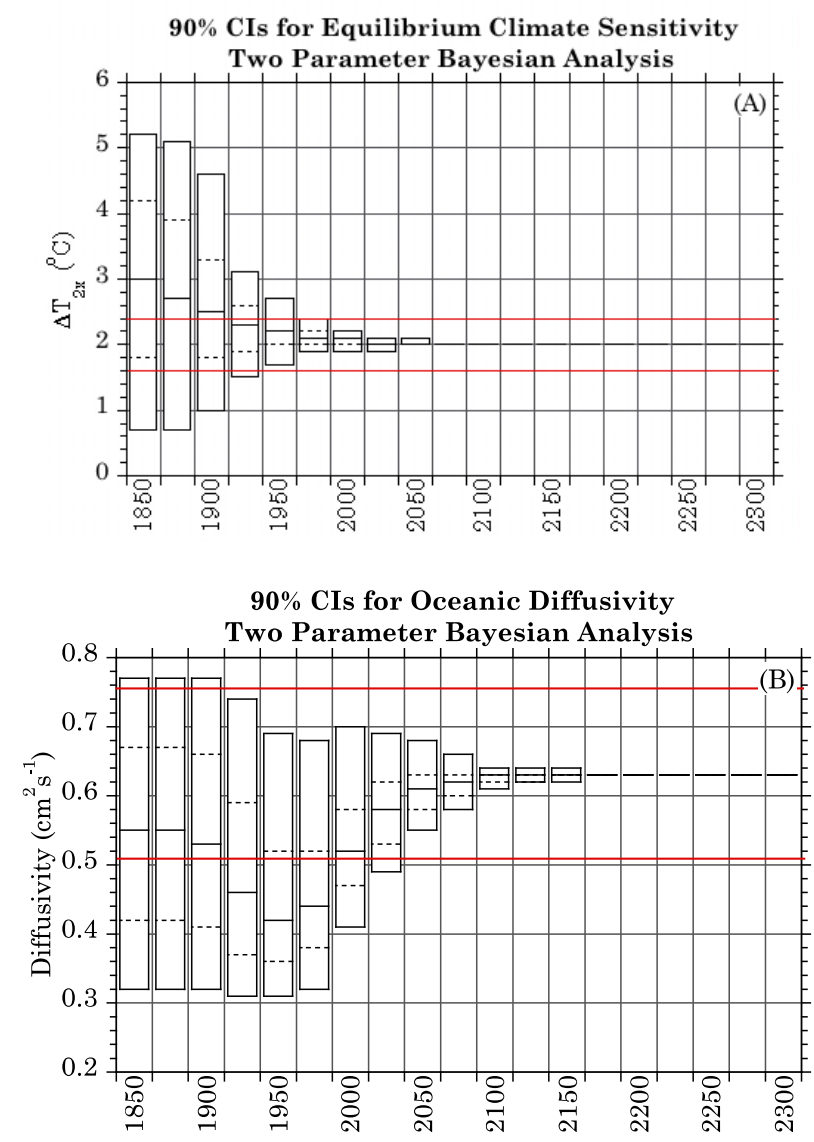

Figure 4. 90 percent confidence intervals for (A) $\Delta T_{2 x}$ and (B) $\boldsymbol{\kappa}$ using Bayesian estimation technique, considering the two variables jointly. "True" values are $2.0^{\circ} \mathrm{C}$ and 0.63 $\mathrm{cm}^{2} \cdot \mathrm{s}^{-1}$, respectively. 

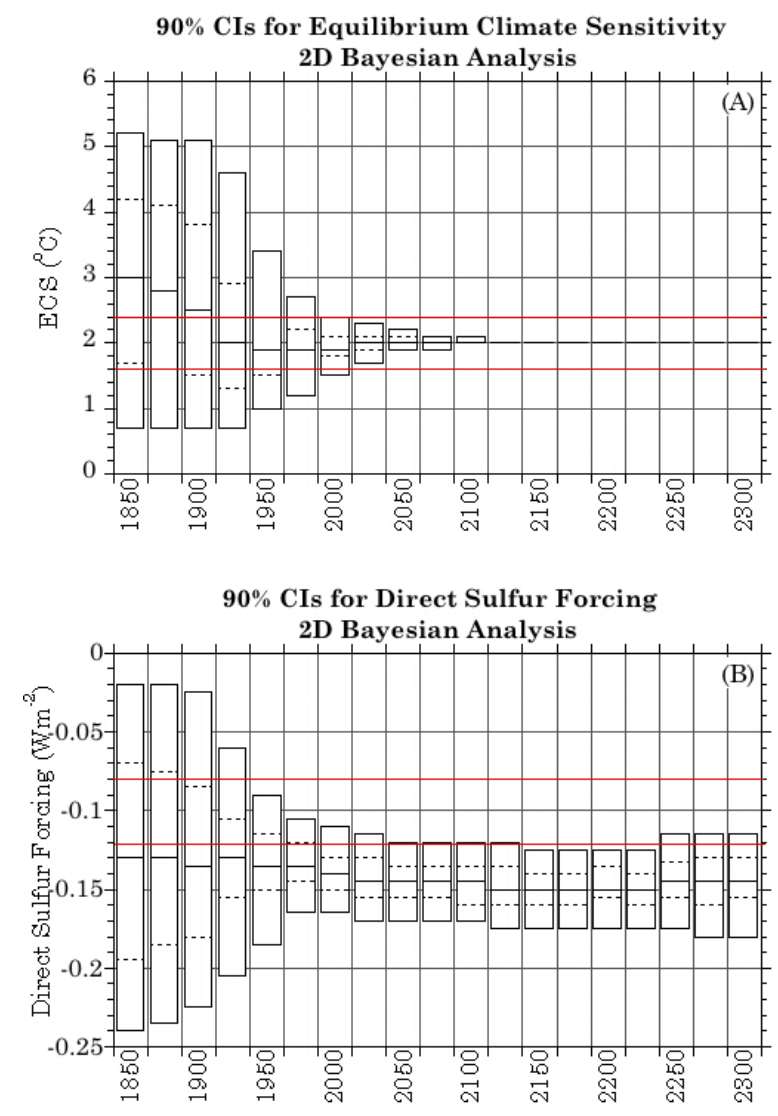

Figure 5. As in Figure 4, but for (A) $\Delta T_{2 x}$ and (B) the direct portion of the sulfate aerosol forcing, considering the two variables jointly. "True" values are $2.0^{\circ} \mathrm{C}$ and $-0.1 \mathrm{Wm}^{-2}$, respectively.

radiative forcing- $12 \mathrm{Wm}^{-2}$ at its peak. Since, by the nature of its lifetime in the atmosphere, the radiative forcing contributed by $\mathrm{CO}_{2}$ and other long-lived greenhouse gases is global in nature, the global-mean temperature and global oceanic heat uptake both increase strongly as the atmosphere and ocean warm. Given the strong, lasting global signal, the Bayesian estimation technique is able to properly learn the "true" values of $\Delta \mathrm{T}_{2 \mathrm{x}}$ and $\kappa$.

In contrast, the interhemispheric temperature difference record is used to constrain the strength of $\mathrm{F}_{\mathrm{ASA}}$. This is a logical choice, since sulfate aerosol particles have a short resident lifetime in the atmosphere, and in a typical year, over 90 percent of sulfate emissions have been in the Northern Hemisphere [56]. Therefore we would expect to see the cooling effects of sulfate more strongly in the Northern Hemisphere as opposed to the Southern Hemisphere, and therefore a relatively warmer Southern Hemisphere were sulfate emissions to increase.

However, the RCP8.5/ECP8.5 scenario does not feature a continuously increasing sulfate emissions record as it does for the long-lived greenhouse gases. While sulfate emissions increase from the beginning of the Industrial
Revolution to $65.1 \mathrm{Tg} \mathrm{S} / \mathrm{yr}$ in 1980 , thereafter sulfate emissions decline to a final value of $12.9 \mathrm{Tg} / \mathrm{yr}$ in years 2100 and thereafter. While the greenhouse-gas forcing signal increases strongly in the 21 st century under this scenario, the sulfate-emissions signal weakens during this period. Without the forcing that would drive a significant temperature difference between Northern and Southern Hemispheres, the opportunity for learning about $\mathrm{F}_{\mathrm{ASA}}$ is reduced.

We tested this hypothesis by substituting an artificial, continuously increasing sulfate forcing record in place of the RCP8.5/ECP8.5 scenario. The Bayesian estimation of $\mathrm{F}_{\mathrm{ASA}}$ is greatly improved using the artificial record. Hence the problem in estimating $\mathrm{F}_{\mathrm{ASA}}$ is a signal-tonoise-ratio problem - the sulfate emissions are not strong enough, for long enough, to create a trend in the interhemispheric temperature difference from which the value of $\mathrm{F}_{\mathrm{ASA}}$ can be constrained. It is worth noting that while the RCP8.5 scenario shows decreasing sulfate emissions in the first decade of the 21 st century, rising sulfate emissions have been observed during that period [56], due largely to industrial pollution from China. If sulfate emissions were to continue to increase in the future, we would expect a more accurate performance from our technique in constraining $\mathrm{F}_{\mathrm{ASA}}$.

Thus far, we have applied the Bayesian estimation technique to synthetic temperature and ocean heat-uptake records. Our next application of this technique will be to use the Bayesian estimation on the instrumental records. Our method will be similar to that outlined in Section 2 above, but instead of comparing to a model simulation chosen as a synthetic dataset we will compare to the observed records.

We may use Bayesian estimation to obtain single-parameter or multi-parameter estimates of each of the climate parameters. There will be some complications in so doing, however. We have already shown above the difficulty in estimating $\mathrm{F}_{\mathrm{ASA}}$ using the Bayesian techniques. An alternate method is, rather than using Bayesian estimation to determine $\mathrm{F}_{\mathrm{ASA}}$, to instead use the SCM's optimized value of this quantity and then use Bayesian analysis to learn $\Delta \mathrm{T}_{2 \mathrm{x}}$ and $\kappa$.

Using the instrumental ocean heat-uptake record creates an additional problem, as the record [8] extends only as far back as 1955. For the cases above, where one of the synthetic observations for known values of the climate parameters was chosen as "truth", this did not present a problem, as no optimization was done at all for those cases, and a synthetic record was taken as the basis for comparison. Here, as there is no oceanic data to which to compare until 1955 , we will carry forward our prescribed prior for $\kappa$ at each time step from 1850 or 1880 , depending on the start of the particular temperature dataset in use. This means that prior to 1955, the analysis 
will essentially be one-parameter. Because there are fewer than 70 years of data in [8], we hypothesize that the Bayesian learning of $\kappa$ will be less than that of $\Delta \mathrm{T}_{2 \mathrm{x}}$ for which much longer datasets are available [3-7]. Nonetheless it is important to learn the extent to which Bayesian learning can shrink the range of $\kappa$ for the period for which we do have data.

Additionally, the SCM may be used to generate future simulated observations based on the current estimations of the values of $\Delta \mathrm{T}_{2 \mathrm{x}}, \mathrm{F}_{\mathrm{ASA}}$, and $\kappa$, which will allow us to test future leaning of the parameters' values. Since we will have a range of values at present day, we can try running the model for different choices of these parameters to test the effect on the Bayesian estimation. Additionally, we can test the effects of a choice of different future emissions scenario on the rate of learning. It is logical to use the other three members of the RCP scenario family-the RCP2.6 [57], RCP4.5 [58], and RCP 6.0 [59] scenarios. We expect that our Bayesian estimations will converge more slowly under the influence of the scenarios with smaller emissions as compared to RCP8.5.

\section{Acknowledgements}

This work was funded by the United States National Science Foundation grant ATM 08-06155. Any opinions, findings, and conclusions or recommendations expressed in this material are those of the authors and do not necessarily reflect the views of the National Science Foundation.

\section{REFERENCES}

[1] M. J. Ring, D. Lindner, E. F. Cross and M. E. Schlesinger, "Causes of the Global Warming Observed Since the 19th Century," Atmospheric and Climate Sciences, 2012, in Press.

[2] M. E. Schlesinger, N. G. Andronova, B. Entwistle, A. Ghanem, N. Ramankutty, W. Wang and F. Yang, "Modeling and Simulation of Climate and Climate Change," In: G. Cini Castagnoli and A. Provenzale, Eds., Past and Present Variability of the Solar-Terrestrial System: Measurement, Data Analysis and Theoretical Models. Proceedings of the International School of Physics "Enrico Fermi" CXXXIII, IOS Press, Amsterdam, 1997, pp. 389-429.

[3] C. P. Morice, J. J. Kennedy, N. A. Rayner and P. D. Jones, "Quantifying Uncertainties in Global and Regional Temperature Change Using an Ensemble of Observational Estimates: The HadCRUT4 Dataset," Journal of Geophysical Research, Vol. 117, 2012, Article ID: D08101. doi:10.1029/2011JD017187

[4] J. Hansen, R. Ruedy, M. Sato and K. Lo, "Global Surface Temperature Change," Reviews of Geophysics, Vol. 48, 2010, Article ID: RG4004. doi:10.1029/2010RG000345.

[5] T. M. Smith, R. W. Reynolds, T. C. Peterson and J. H.
Lawrimore, "Improvements to NOAA's Historical Merged Land-Ocean Surface Temperature Analysis," Journal of Climate, Vol. 21, No. 10, 2008, pp. 2283-2296. doi:10.1175/2007JCLI2100.1.

[6] K. Ishihara, "Calculation of Global Surface Temperature Anomalies with COBE-SST (Japanese)," Weather Service Bulletin, Vol. 73, 2006, pp. S19-S25.

[7] K. Ishihara, "Estimation of Standard Errors in Global Average Surface Temperature (Japanese)," Weather Service Bulletin, Vol. 74, 2007, pp. 19-26.

[8] S. Levitus, J. I. Antonov, T. P. Boyer, R. A. Locarnini, H. E. Garcia and A. V. Mishonov, "Global Ocean Heat Content 1955-2008 in Light of Recently Revealed Instrumentation Problems," Geophysical Resarch Letters, Vol. 36, 2009, Article ID: L07608. doi:10.1029/2008GL037155.

[9] D. Lindner, "Characterization of the Modes of Interannual-to-Centennial Variability in Observed Near-Surface Temperatures," Master Thesis, University of Illinois at Urbana-Champaign, Urbana, 2010.

[10] N. G. Andronova and M. E. Schlesinger, "Objective Estimation of the Probability Density Function for Climate Sensitivity," Journal of Geophysical Research, Vol. 106, No. D19, 2001, pp. 22605-22611. doi:10.1029/2000JD000259.

[11] A. R. Solow, "Bootstrapping Correlated Data," Mathematical Geology, Vol. 17, No. 7, 1985, pp. 769-775. doi:10.1007/BF01031616.

[12] A. Neumeier and T. Schneider, "Estimation of Parameters and Eigenmodes of Multivariate Autoregressive Models," ACM Transactions on Mathematical Software, Vol. 27, 2001, pp. 27-57.

[13] T. Schneider and A. Neumeier, "Algorithm 808: ARFitA MATLAB Package for the Estimation of Parameters and Eigenmodes of Multivariate Autogressive Models," ACM Transactions on Mathematical Software, Vol. 27, 2001, pp. 58-65.

[14] E. S. Epstein, "Statistical Inference and Prediction in Climatology: A Bayesian Approach," American Meteorological Society, 1985

[15] P.-S. Chu and X. Zhao, "Bayesian Analysis for Extreme Climatic Events: A Review," Atmospheric Research, Vol. 102, No. 3, 2011, pp. 243-262. doi:10.1016/j.atmosres.2011.07.001.

[16] C. E. Forest, P. H. Stone, A. P. Sokolov, M. R. Allen and M. D. Webster, "Quantifying Uncertainties in Climate System Properties with the Use of Recent Climate Observations," Science, Vol. 295, No. 5552, 2002, pp. 113117. doi:10.1126/science.1064419.

[17] C. E. Forest, P. H. Stone and A. P. Sokolov, "Estimated PDFs of Climate System Properties Including Natural and Anthropogenic Forcings," Geophysical Research Letters, Vol. 33, 2006, Article ID: L01705. doi:10.1029/2005GL023977.

[18] L. Tomassini, P. Reichert, R. Knutti, T. F. Stocker and M. E. Borsuk, "Robust Bayesian Uncertainty Analysis of Climate System Properties Using Markov Chain Monte Carlo Methods," Journal of Climate, Vol. 20, No. 7, 2007, pp. 1239-1254. doi:10.1175/JCLI4064.1. 
[19] C. E. Forest, P. H. Stone and A. P. Sokolov, "Constraining Climate Model Parameters from Observed 20th Century Changes," Tellus A, Vol. 60, No. 5, 2008, pp. 911920. doi:10.1111/j.1600-0870.2008.00346.x.

[20] J. D. Annan and J. C. Hargraves, "On the Generation and Interpretation of Probabilistic Estimates of Climate Sensitivity," Climatic Change, Vol. 104, No. 3-4, 2011, pp. 423-436. doi:10.1007/s10584-009-9715-y.

[21] S. S. Leroy, "Detecting Climate Signals: Some Bayesian Aspects," Journal of Climate, Vol. 11, No. 4, 1998, pp. 640-651. doi:10.1175/1520-0442(1998)011<0640:DCSSBA >2.0.C $\mathrm{O} ; 2$.

[22] T. C. K. Lee, F. W. Zwiers, G. C. Hegerl, X. Zhang and M. Tsao, "A Bayesian Climate Change Detection and Attribution Assessment," Journal of Climate, Vol. 18, No. 13, 2006, pp. 2429-2440. doi:10.1175/JCLI3402.1.

[23] S. S. Leroy and J. G. Anderson, "Optimal Detection of Regional Trends Using Global Data," Journal of Climate, Vol. 23, No. 16, 2010, pp. 4438-4446. doi:10.1175/2010JCLI3550.1.

[24] L. Tomassini, R. Knutti, G.-K. Plattner, D. P. van Vuuren, T. F. Stocker, R. B. Howarth and M. E. Borsuk, "Uncertainty and Risk in Climate Projections for the 21st Century: Comparing Mitigation to Non-Intervention Scenarios," Climatic Change, Vol. 103, No. 3-4, 2010, pp. 399422. doi:10.1007/s10584-009-9763-3.

[25] S.-K. Min and A. Hense, "A Bayesian Assessment of Climate Change Using Multi-Model Ensembles. Part I: Global Mean Surface Temperature," Journal of Climate, Vol. 19, No. 13, 2006, pp. 3237-3256. doi:10.1175/JCLI3784.1.

[26] S.-K. Min, D. Simonis and A. Hense, "Probabilistic Climate Change Predictions Applying Bayesian Model Averaging," Philosophical Transactions of the Royal Society A, Vol. 365, No. 1857, 2007, pp. 2103-2116. doi:10.1098/rsta.2007.2070.

[27] R. L. Smith, C. Tebaldi, D. Nychka and L. O. Mearns, "Bayesian Modeling of Uncertainty in Ensembles of Climate Models," Journal of the American Statistical Association, Vol. 104, No. 485, 2009, pp. 97-116. doi:10.1198/jasa.2009.0007.

[28] R. Furrer, R. Knutti, S. R. Sain, D. W. Nychka and G. A. Meehl, "Spatial Patterns of Probabilistic Temperature Change Projections from a Multivariate Bayesian Analysis," Geophysical Research Letters, Vol. 34, 2006, Article ID: L06711. doi:10.1029/2006GL027754.

[29] R. Furrer, S. R. Sain, D. Nychka and G. A. Meehl, "Multivariate Bayesian Analysis of Atmosphere-Ocean General Circulation Models," Environmental and Ecological Statistics, Vol. 14, No. 3, 2007, pp. 249-266. doi:10.1007/s10651-007-0018-Z.

[30] C. Tebaldi and B. Sanso, "Joint Projections of Temperature and Precipitation Change from Multiple Climate Models: A Hierarchical Bayesian Approach," Journal of the Royal Statistical Society A, Vol. 172, No. 1, 2009, pp. 83-106. doi:10.1111/j.1467-985X.2008.00545.x.

[31] F. Beltran, B. Sanso, R. Lemos and R. Mendelssohn, "Joint Projections of North Pacific Sea Surface Tempera- ture from Different Global Climate Models," University of California, Santa Cruz, 2011.

[32] C. Tebaldi, L. O. Mearns, D. Nychka and R. W. Smith, "Regional Probabilities of Precipitation Change: A Bayesian Analysis of Multimodel Simulations," Geophysical Research Letters, Vol. 31, 2004, Article ID: L24213.

[33] C. Tebaldi, R. W. Smith, D. Nychka and L. O. Mearns, "Quantifying Uncertainty in Projections of Regional Climate Change: A Bayesian Approach to the Analysis of Multimodel Ensembles," Journal of Climate, Vol. 18, No. 10, 2005, pp. 1524-1540. doi:10.1175/JCLI3363.1.

[34] S.-K. Min and A. Hense, "A Bayesian Assessment of Climate Change Using Multi-Model Ensembles. Part II: Regional and Seasonal Mean Surface Temepratures," Journal of Climate, Vol. 20, No. 12, 2007, pp. 2769-2790. doi:10.1175/JCLI4178.1.

[35] J. B. Elsner and B. H. Bossak, "Bayesian Analysis of US Hurricane Climate," Journal of Climate, Vol. 14, No. 23, 2001, pp. 4341-4350. doi:10.1175/1520-0442(2001)014<4341:BAOUSH $>2.0 . \mathrm{C}$ $\underline{\mathrm{O} ; 2 .}$

[36] P.-S. Chu and X. Zhao, "Bayesian Change-Point Analysis of Tropical Cyclone Activity: The Central North Pacific case," Journal of Climate, Vol. 17, No. 24, 2004, pp. 4893-4901. doi: 10.1175/JCLI-3248.1.

[37] J. B. Elsner and T. H. Jagger, "Prediction Models for Annual US Hurricane Counts," Journal of Climate, Vol. 19, No. 12, 2006, pp. 2935-2952. doi:10.1175/JCLI3729.1.

[38] X. Zhao and P.-S. Chu, "Bayesian Multiple Changepoint Analysis of Hurricane Activity in the Eastern North Pacific: A Markov Chain Monte Carlo Approach," Journal of Climate, Vol. 19, No. 4, 2006, pp. 564-578. doi:10.1175/JCLI3628.1.

[39] S. J. Camargo, A. W. Robertson, S. J. Gaffney, P. Smyth and M. Ghil, "Cluster Analysis of Typhoon Tracks. Part I: General Properties," Journal of Climate, Vol. 20, No. 14, 2007, pp. 3635-3653. doi:10.1175/JCLI4188.1.

[40] P.-S. Chu and X. Zhao, "A Bayesian Regression Approach for Predicting Seasonal Tropical Cyclone Activity over the Central North Pacific," Journal of Climate, Vol. 20, No. 15, 2007, pp. 4002-4013. doi:10.1175/JCLI4214.1.

[41] C.-H. Ho, H. S. Kim, P.-S. Chu and J.-H. Kim, "Seasonal Prediction of Tropical Cyclone Frequency over the East China Sea through a Bayesian Poisson-Regression Method," Asia-Pacific Journal of Atmospheric Sciences, Vol. 45, No. 1, 2009, pp. 45-54.

[42] J.-Y. Tu, C. Chou and P.-S. Chu, "The Abrupt Shift of Typhoon Activity in the Vicinity of Taiwan and Its Association with Western North Pacific-East Asian Climate Change," Journal of Climate, Vol. 22, No. 13, 2009, pp. 3617-3628. doi:10.1175/2009JCLI2411.1.

[43] S. S. Chand, K. J. E. Walsh and J. C. L. Chan, “A Bayesian Regression Approach to Seasonal Prediction of Tropical Cyclones Affecting the Fiji Region," Journal of Climate, Vol. 23, No. 13, 2010, pp. 3425-3445. doi:10.1175/2010JCLI3521.1.

[44] P.-S. Chu, X. Zhao, C.-H. Ho, H.-S. Kim, M.-M. Lu and 
J.-H. Kim, "Bayesian Forecasting of Seasonal Typhoon Activity: A Track Pattern-Oriented Categorization Approach," Journal of Climate, Vol. 23, No. 24, 2010, pp. 6654-6668. doi:10.1175/2010JCLI3710.1.

[45] T. H. Jagger and J. B. Elsner, "A Consensus Model for Seasonal Hurricane Prediction," Journal of Climate, Vol. 23, No. 22, 2009, pp. 6090-6099. doi:10.1175/2010JCLI3686.1.

[46] M.-M. Lu, P.-S. Chu and Y.-C. Lin, "Seasonal Prediction of Tropical Cyclone Activity in the Vicinty of Taiwan Using the Bayesian Multivariate Regression Method," Weather and Forecasting, Vol. 25, No. 6, 2010, pp. 17801795. doi:10.1175/2010WAF2222408.1.

[47] M. Kallache, E. Maksimovich, P.-A. Michelangeli and P. Naveau, "Multi-Model Combination by a Bayesian Hierarchical Model: Assessment of Ice Accumulation over the Oceanic Arctic Region," Journal of Climate, Vol. 23, No. 20, 2010, pp. 5421-5436. doi:10.1175/2010JCLI3107.1.

[48] B. F. Hobbs, "Bayesian Methods for Analysing Climate Change and Water Resource Uncertainties," Journal of Environmental Management, Vol. 49, No. 1, 1997, pp. 53-72. doi:10.1006/jema.1996.0116,

[49] L. Perreault, J. Bernier, B. Bobee and E. Parent, "Bayesian Change-Point Analysis in Hydrometeorological Time Series. Part 1: The Normal Model Revisited," Journal of Hydrology, Vol. 235, No. 3-4, 2000, pp. 221-241. doi:10.1016/S0022-1694(00)00270-5.

[50] L. Perreault, J. Bernier, B. Bobee and E. Parent, "Bayesian Change-Point Analysis in Hydrometeorological Time Series. Part 2: Comparison of Change-Point Models and Forecasting," Journal of Hydrology, Vol. 235, No. 3-4, 2000, pp. 242-263. doi:10.1016/S0022-1694(00)00271-7.

[51] B. Renard, M. Land and P. Bois, "Statistical Analysis of Extreme Events in A Non-Stationary Context via a Bayesian Framework: Case Study with Peak-over-Threshold Data," Stochastic Environmental Research and Risk Assessment, Vol. 21, No. 2, 2006, pp. 97-112. doi:10.1007/s00477-006-0047-4.
[52] C. H. R. Lima and U. Lall, "Spatial Scaling in a Changing Climate: A Hierarchical Bayesian Model for Non-Stationary Multi-Site Annual Maximum and Monthly Streamflow," Journal of Hydrology, Vol. 383, No. 3-4, 2010, pp. 307-318. doi:10.1016/j.jhydrol.2009.12.045.

[53] D. Peavoy and C. Franzke, "Bayesian Analysis of Rapid Climate Change during the Last Glacial Using Greenland Delta O-18 Data," Climate of the Past, Vol. 6, No. 6, pp. 787-794. doi:10.5194/cp-6-787-2010.

[54] K. Riahi, et al., "RCP8.5-A Scenario of Comparatively High Greenhouse Emissions," Climatic Change, Vol. 109, No. 1-2, 2011, pp. 33-57. doi:10.1007/s10584-011-0149-y.

[55] M. Ghil, M. R. Allen, M. D. Dettinger, K. Ide, D. Kondrashov, M. E. Mann, A. W. Robertson, A. Saunders, Y. Tian, F. Varadi and P. Yiou, "Advanced Spectral Methods for Climatic Time Series," Reviews of Geophysics, Vol. 40, No. 1, 2002, Article ID: 1003. doi:10.1029/2000RG000092.

[56] S. J. Smith, J. van Aardenne, Z. Klimont, R. Andres, A. C. Volke and S. Delgado Arias, "Anthropogenic Sulfur Dioxide Emissions 1850-2005," Atmospheric Chemistry and Physics, Vol. 11, 2011, pp. 1101-1116. doi:10.5194/acp-11-1101-2011.

[57] D. P. van Vurren, et al., "RCP2.6: Exploring the Possibility to Keep Global Mean Temperature Increase below $2^{\circ} \mathrm{C}$," Climatic Change, Vol. 109, No. 1-2, 2011, pp. 95116. doi:10.1007/s10584-011-0152-3.

[58] A. M. Thomson, et al., "RCP4.5: A Pathway for Stabilization of Radiative Forcing by 2100," Climatic Change, Vol. 109, No. 1-2, 2011, pp. 77-94. doi:10.1007/s10584-011-0151-4.

[59] T. Masui, et al., "An Emission Pathway for Stabilization at $6 \mathrm{Wm}^{-2}$ Radiative Forcing," Climatic Change, Vol. 109, No. 1-2, 2011, pp. 59-76. doi:10.1007/s10584-011-0150-5. 